\title{
An Unusual Case of Acute Abdomen: Ovarian Vein Thrombosis
}

Asmita Ghimire, ${ }^{1}$ Meeta Singh, ${ }^{1}$ Kesang Dikki Bista, ${ }^{1}$ Neebha Ojha, ${ }^{1}$ Sunita Bajracharya, ${ }^{1}$ Neeta Katuwal ${ }^{1}$

'Department of Obstetrics and Gynaecology, Maharajgunj Medical Campus, Maharajgunj,Kathmandu, Nepal.

\section{ABSTRACT}

Ovarian vein thrombosis is one of the rare causes for acute abdomen. Occult presentation of this disease may lead to diagnostic dilemma. A sixteen years female who was operated as a case of ectopic pregnancy was finally diagnosed as having ovarian vein thrombosis. She was successfully treated with anticoagulant

Keywords: Anticoagulants; acute abdomen; ovarian vein thrombosis.

\section{INTRODUCTION}

Ovarian vein thrombosis (OVT) is a rare condition occurring mainly in the postpartum period often leading to fatal complications like sepsis, pulmonary embolism, thrombosis of inferior venacava and renal veins if misdiagnosed. Typical presentation is fever, lower abdominal pain and pelvic mass, with incidence of $1 / 600$ to $1 / 2000$ pregnancy. ${ }^{1,2}$ Few have atypical and silent presentation. It is associated with conditions like inflammatory bowel disease, malignancies, abdomino-pelvic surgery, post abortal sepsis and pelvic inflammatory diseases and mimics endometritis, acute pyelonephritis and acute appendicitis. Idiopathic OVT, a very rare event has been described only through case reports in literature. Here is a unique case of OVT successfully managed in TU teaching hospital.

\section{CASE REPORT}

A 16 years girl who had undergone termination of pregnancy by medical methods in local medical shop in Kathmandu at two and a half months was admitted at Tribhuwan University Teaching Hospital with complaints of lower abdominal pain and vaginal spotting for four days. No prior history of instrumentation or surgical procedures was there. She was hemodynamically stable. On per abdomen examination uterus was enlarged to 16 weeks size and per speculum examination revealed blood smeared vagina. Cervical motion tenderness was positive. Her hemoglobin was $10.5 \mathrm{gm} \%$. Ultrasonogram (USG) showed bulky uterus with empty cavity with free fluid in Morrison's pouch and pelvis. Diagnostic aspiration revealed blood mixed fluid. UPT was positive. On suspicion emergency laparotomy was done for ruptured ectopic pregnancy with fibroid uterus as uterus was enlarged to 16 weeks size. Intraoperatively, dark blue discoloration of uterus and fallopian tubes and mildly enlarged congested ovaries were seen. Surrounding structures appeared healthy. Around $100 \mathrm{ml}$ of serosanginous fluid was present in the peritoneal cavity. Abdomen was closed after peritoneal washing. The tissue obtained on check curettage done on the same setting showed products of conception on histopathological examination.

She was started on intravenous antibiotics, septic profile, coagulation profile and immunological profile (ANA, dsDNA and cardiolipin antibody) were sent the very next day and came out to be normal except her $\mathrm{Hb}$ which dropped to $8.6 \mathrm{gm} \%$. USG Doppler showed bilateral enlarged ovaries with hypo-echoic appearance and no stromal flow. CT scan showed enlarged ovaries without any blood flow.

After cardiothoracic and vascular consultation with diagnosis of OVT, Tab warfarin $5 \mathrm{mg}$ po od along with Inj. Heparin $40 \mathrm{mg} \mathrm{s} / \mathrm{c}$ bd was started on and regular PT/INR monitoring was done. She had fever and mild abdominal pain on and off which was relieved by analgesics and antipyretics. On $5^{\text {th }}$ POD her INR raised to 4.8 so anticoagulants were stopped. As her hemoglobin dropped to 7.7 two pints blood transfusion was given. Intravenous Antibiotics were given for a week and then switched to oral medications. Observation with regular PT/INR monitoring was done. On POD13 after INR level

Correspondence: Asmita Ghimire, Department of Obstetrics and Gynaecology, Maharajguni Medical Campus, Maharaiguni, Kathmandu, Nepal. Email: asmitaghimire4@gmail.com, Phone: +9779875011926. 
normalized. Oral anticoagulant was restarted and readjusted in dose of $4 \mathrm{mg}$ po OD. UPT done on POD17 was negative. USG doppler on POD17 confirmed flow to both ovaries. On POD 20 during discharge she was readjusted on warfarin $2 \mathrm{mg}$ dose and asked to follow up in OPD with Doppler reports.

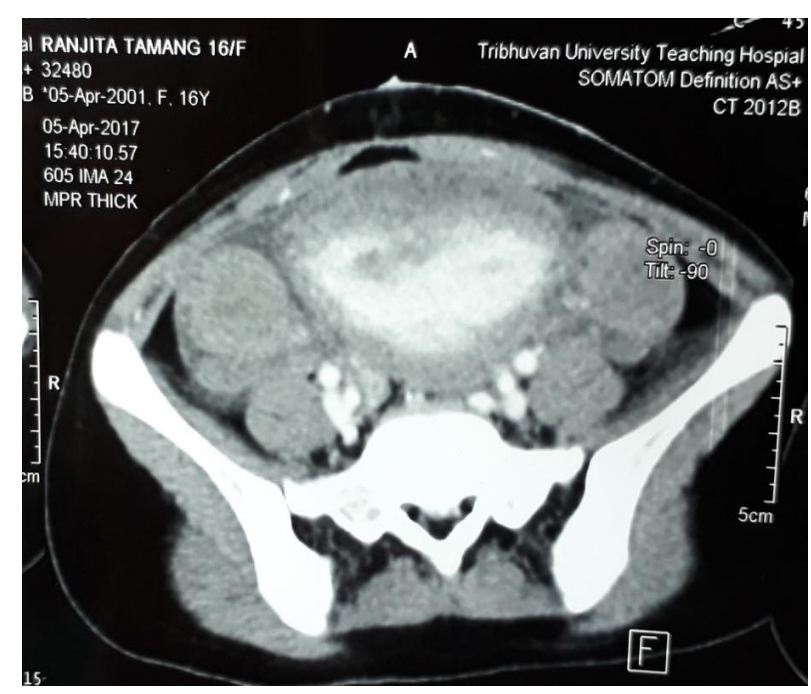

Fig. Contrast CT Scan showing no any blood flow to ovaries.

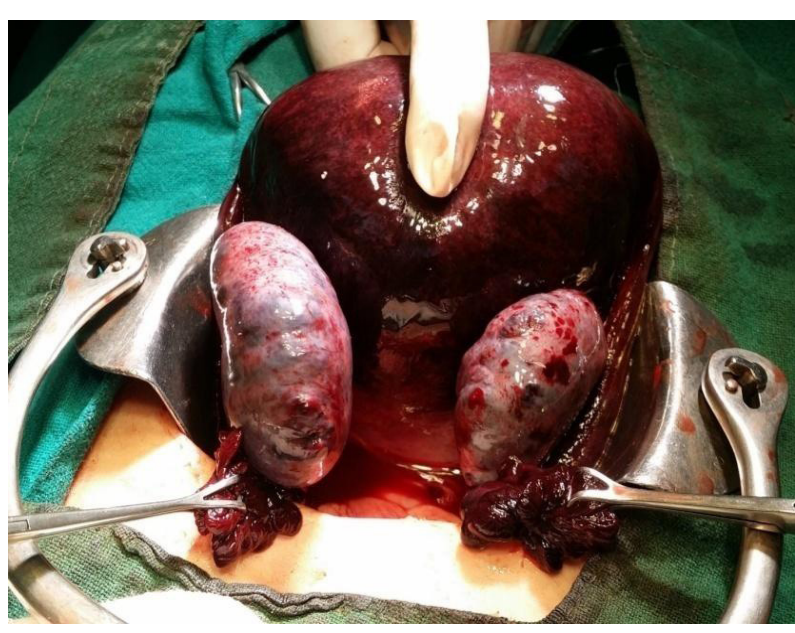

Fig. Fig: Intra operative picture showing discolored Uterus, tubes and Ovaries.

\section{DISCUSSION}

Ovarian Vein Thrombosis is a rare potentially life threatening condition. It was first described by Austin in $1956^{3}$. It occurs more on the right side due to right ovarian vein being larger than left and lack of competent valves, incidence ranging from $70-90 \%$. Bilateral OVT is seen in $11-14 \% .^{4}$

Common symptoms are fever, nausea, vomiting, chills, lower abdominal pain, tachycardia and flank pain.
Predisposing factors like PID, malignancy, recent surgery, and others have been linked with it. Anti-phospholipid antibody syndrome (APS) is also considered as a causative agent which is characterized by recurrent vascular thrombosis, pregnancy morbidity. ${ }^{5}$ But Screening study for APS was not performed in our case because this was her first thrombotic event. Till date only 5 cases of idiopathic OVT has been reported. ${ }^{6}$ Differential diagnosis includes adnexal torsion, ectopic pregnancy, tubo-ovarian abscess, intussusception, appendicitis, peritonitis and pyelonephritis.

USG, CT scan and MRI help in confirming the diagnosis. ${ }^{7}$ Findings of ultrasound are anechoic to hypo-echoic mass between the adnexa and absence of blood flow within the mass. Color doppler has sensitivity $55.6 \%$ and specificity $41.5 \%$. A suspicious USG should be followed by CT scan whose sensitivity is $77.8 \%$ and specificity $62.5 \%$. MRI has the highest sensitivity of $100 \% .{ }^{7}$ CT scan is the preferred diagnostic modality. In our case Doppler and CT scan both showed absence of blood flow to the ovaries.

Spontaneous resolution can occur in some but due to fear of catastrophic events anticoagulant is required. Anticoagulants shrink small septic emboli and hasten fever resolution. Treatment consists of 5,000 U intravenous bolus heparin followed by an initial maintenance dose of $1,000 \mathrm{U} / \mathrm{hr}$ or 10,000 units daily ${ }^{8}$ with aim of achieving APTT of 1.5 to 2 times normal.

Resolution has been documented after 7 to 14 days of therapy. Recommended period is 3 to 6 months anticoagulation treatment and 7 to 10 days course of antibiotics until there is radiologically confirmed resolution of the thrombus. ${ }^{7}$

Interestingly, the patient's pain improved on anticoagulation despite imaging findings demonstrating no change in the size of the thrombus. Heparin has anti-inflammatory properties that exist apart from its role in anticoagulation. It inhibits mediators involved in inflammation. ${ }^{10}$ We had started our patient on intravenous and oral anticoagulants and finally kept on her oral anticoagulants which proved to be successful showing regain of blood supply to ovaries as evidenced by Doppler in a duration of 2 weeks. Surgical modalities of treatment are IVC filter and ovarian vein ligation.

Recurrence rate of OVT appears to be low, with only 3 recurrent venous thrombi per 100 patient-years. ${ }^{9}$

\section{CONCLUSIONS}

Ovarian vein thrombosis, though a rare presentation should be a differential diagnosis for women presenting 
with acute abdomen, fever and mass per abdomen. Proper diagnosis and timely management can prevent catastrophic complications. Our case thus highlights the importance of considering the rare diagnosis and the diagnostic and therapeutic options for this disease.

\section{REFERENCES}

1. Dunnihoo DR, Gallaspy JW, WISE RB, Otterson WN. Postpartum ovarian vein thrombophlebitis: a review. Obstet Gynecol Surv. 1991;46(7):415-27.[Full Text]

2. Royo P, Alonso-Burgos A, García-Manero M, Lecumberri R, Alcázar JL. Postpartum ovarian vein thrombosis after cesarean delivery: a case report. J Med Case Rep. 2008;2(1):105.[Full Text]

3. Austin OG. Massive thrombophlebitis of the ovarian vein: a case report. Am J Obstet Gynecol. 1956;72(2):428-9.

4. Baran GW, Frisch KM. Duplex Doppler evaluation of puerperal ovarian vein thrombosis. Am J Roentgenol. 1987;149(2):321-2.[Full Text]

5. Amengual O, Atsumi T, Koike T. Pathophysiology of thrombosis and potential targeted therapies in anti-phospholipid syndrome. Curr Vasc Pharmacol. 2011;9(5):606-18.[Full Text]
6. Harris K, Mehta S, Iskhakov E, Chalhoub M, Maniatis T, Forte F, Alkaied H. Ovarian vein thrombosis in the nonpregnant woman: an overlooked diagnosis. Ther Adv Hematol. 2012 Oct;3(5):325-8.[Full Text]

7. Dessole S, Capobianco G, Arru A, Demurtas P, Ambrosini G. Postpartum ovarian vein thrombosis: an unpredictable event: two case reports and review of the literature. Arch Gynecol Obstet. 2003;267(4):242-6.[Full Text]

8. Pineo GF, Hull RD. Heparin and low-molecular-weight heparin in the treatment of venous thromboembolism. Baillieres Clin Haematol. 1998;11:621-37.

9. Wysokinska EM, Hodge D, McBane II RD. Ovarian vein thrombosis: incidence of recurrent venous thromboembolism and survival. Thromb Haemost. 2006;96(02):126-31.[Full Text]

10. Cassinelli G, Naggi A. Old and new applications of nonanticoagulant heparin. Int J Cardiol. 2016;212:S14-21. [Science Direct] 